\title{
Submillimeter emission from water in the W3 region ${ }^{\star}$
}

\author{
C. D. Wilson ${ }^{1}$, A. Mason ${ }^{1}$, E. Gregersen ${ }^{1}$, A. O. H. Olofsson ${ }^{2}$, P. Bergman ${ }^{2}$, R. Booth ${ }^{2}$, N. Boudet ${ }^{3}$, V. Buat ${ }^{4}$,
} C. L. Curry ${ }^{5}$, P. Encrenaz ${ }^{6}$, E. Falgarone ${ }^{7}$, P. Feldman ${ }^{8}$, M. Fich ${ }^{5}$, H. G. Floren ${ }^{9}$, U. Frisk ${ }^{10}$, M. Gerin ${ }^{7}$, J. Harju ${ }^{11}$, T. Hasegawa ${ }^{12}$, A. Hjalmarson ${ }^{2}$, M. Juvela ${ }^{11}$, S. Kwok ${ }^{12}$, B. Larsson ${ }^{9}$, A. Lecacheux ${ }^{13}$, T. Liljestrom ${ }^{14}$, R. Liseau $^{9}$,

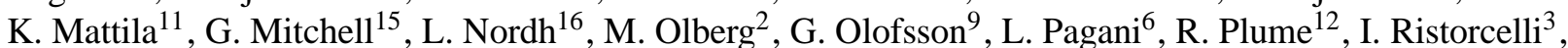
Aa. Sandqvist ${ }^{9}$, G. Serra ${ }^{3}$, N. Tothill ${ }^{15}$, K. Volk ${ }^{12}$, and F. von Scheele ${ }^{10}$

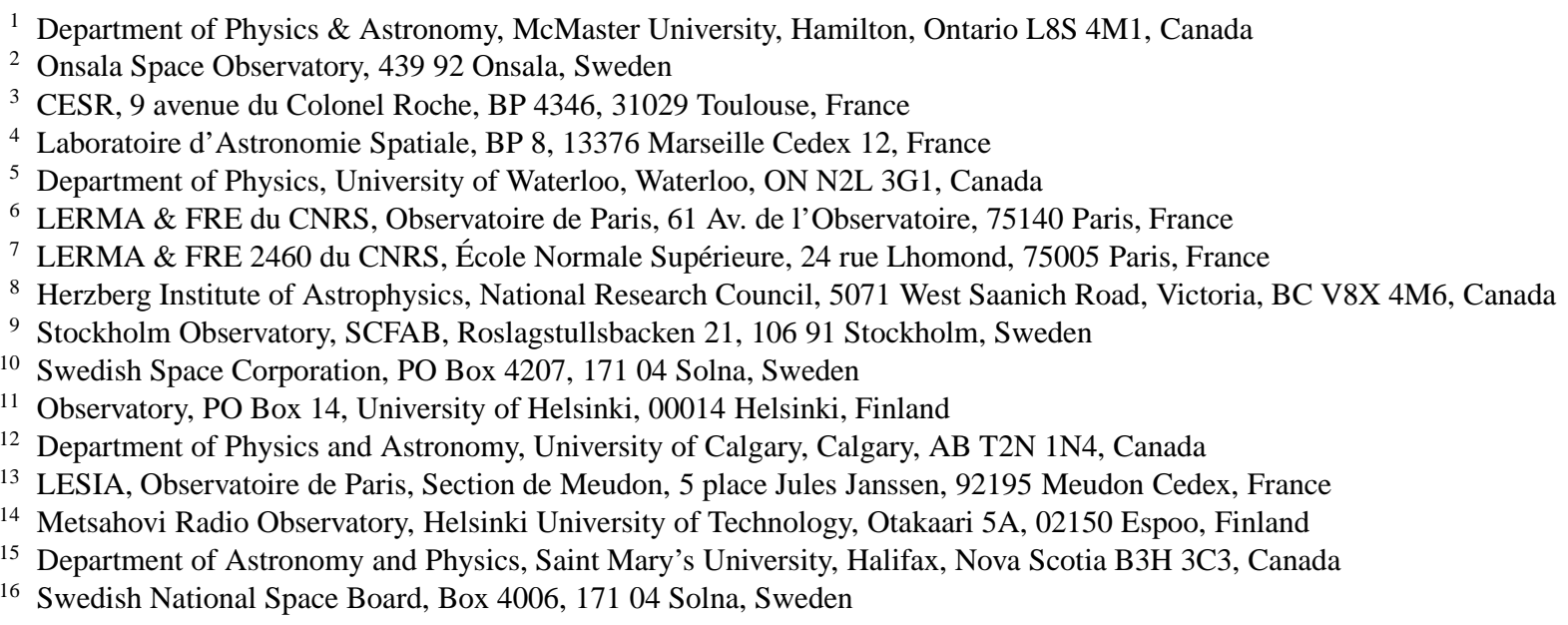

Received 29 November 2002 / Accepted 29 January 2003

\begin{abstract}
Using the Odin satellite, we have mapped the submillimeter emission from the $1_{10}-1_{01}$ transition of ortho-water in the W3 star-forming region. A $5^{\prime} \times 5^{\prime}$ map of the W3 IRS4 and W3 IRS5 region reveals strong water lines at half the positions in the map. The relative strength of the Odin lines compared to previous observations by SWAS suggests that we are seeing water emission from an extended region. Across much of the map the lines are double-peaked, with an absorption feature at $39 \mathrm{~km} \mathrm{~s}^{-1}$; however, some positions in the map show a single strong line at $-43 \mathrm{~km} \mathrm{~s}^{-1}$. We interpret the double-peaked lines as arising from optically thick, self-absorbed water emission near the W3 IRS5, while the narrower blue-shifted lines originate in emission near W3 IRS4. In this model, the unusual appearance of the spectral lines across the map results from a coincidental agreement in velocity between the emission near W3 IRS4 and the blue peak of the more complex lines near W3 IRS5. The strength of the water lines near W3 IRS4 suggests we may be seeing water emission enhanced in a photon-dominated region.
\end{abstract}

Key words. ISM: individual objects: W3 - ISM: molecules - stars: formation

\section{Introduction}

Water has long been thought to be a potentially important coolant in the molecular interstellar medium (Goldsmith \& Langer 1978). However, recent SWAS observations have

Send offprint requests to: $\mathrm{C}$. D. Wilson, e-mail: wilson@physics.mcmaster.ca

* Based on observations with Odin, a Swedish-led satellite project funded jointly by the Swedish National Space Board (SNSB), the Canadian Space Agency (CSA), the National Technology Agency of Finland (Tekes), and Centre National d'Études Spatiales (CNES). The Swedish Space Corporation was the industrial prime contractor and is also responsible for the satellite operation. shown its gas phase abundance to be significantly lower than expected, a fact which is most likely attributable to freezing out of molecules onto grains (i.e. Bergin et al. 2000). The abundance of water shows significant variations from one region to another, with abundances in hot cores and shock-heated outflows at least 100 times larger than the abundance in the colder material that dominates in molecular clouds (Snell et al. 2000a; Neufeld et al. 2000). In this paper, we present a small map made with the Odin satellite (cf. Frisk et al. 2003; Hjalmarson et al. 2003; Nordh et al. 2003) of extended water emission from the ambient molecular gas in W3 which reveals intriguing variations in the line shape across the region. 


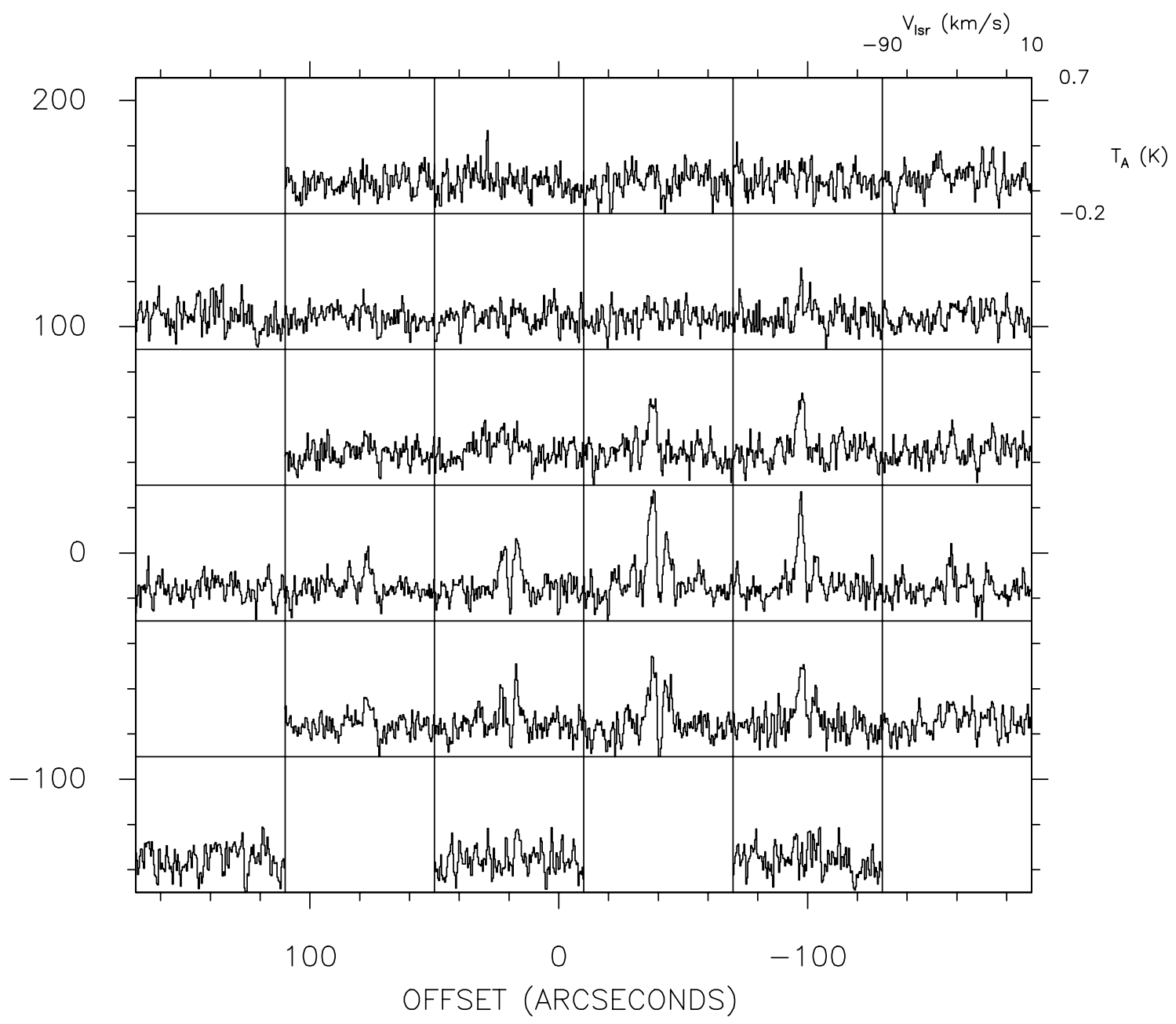

Fig. 1. Individual water spectra observed towards W3 Main. The $(0,0)$ position is located at 02:25:40.5 +62:06:20 (J2000), slightly north of W3 IRS5. The map was obtained on a 1' grid except for the extreme eastern and southern edges, which were observed on a $2^{\prime}$ grid. (For ease of display, spectra on these edges are plotted roughly $\left(20^{\prime \prime}, 20^{\prime \prime}\right)$ from their true position; the true locations of these spectra are shown in Fig. 3.) We estimate the absolute pointing accuracy of the map to be $\sim 15^{\prime \prime}$.

The W3 molecular cloud lies at a distance of roughly $2.3 \mathrm{kpc}$ and contains two well-studied regions of massive star formation, W3 Main and W3(OH). W3 Main contains an infrared cluster (Megeath et al. 1996), which includes the two luminous infrared sources W3 IRS5 and IRS4 (Wynn-Williams et al. 1972), a number of extremely compact HII regions (Tieftrunk et al. 1997), and a powerful outflow (Bally \& Lada 1983; Hasegawa et al. 1994). W3 IRS5 contains numerous water masers which are predominantly associated with two outflows (Imai et al. 2000). Helmich et al. (1994) find that W3 IRS5 is in an earlier evolutionary stage than W3 IRS4, which may be a photon dominated region on the far side of the molecular core (Helmich \& van Dishoeck 1997). The molecular gas in W3 Main is organized into three cores; one core coincides with W3 IRS5, a second core lies just south of W3 IRS4 (i.e. Tieftrunk et al. 1995), and a third core lies south-east of W3 IRS5 (Tieftrunk et al. 1998).

Snell et al. (2000a) detected the $557 \mathrm{GHz}$ line of orthowater towards W3 and other molecular cloud cores with the SWAS satellite and estimate water abundances between $6 \times$ $10^{-10}$ and $1 \times 10^{-8}$. These abundances are at least 100 times smaller than the water abundance estimated using ISO observations of higher energy lines from the very compact hot cores found in some of these regions (i.e. van Dishoeck \& Helmick 1996). In W3, Snell et al. (2000a) find the strong absorption feature in the $\mathrm{H}_{2} \mathrm{O}$ spectrum coincides with the peak of the ${ }^{13} \mathrm{CO} J=5-4$ emission line, which indicates that water emission in W3 is self-absorbed. In this paper, we present a $5^{\prime} \times 5^{\prime}$ fully-sampled map of the water emission from the W3 region with a factor of two better resolution than the SWAS detection. Our map reveals intriguing variations in the shape and strength of the water line (Fig. 1) and suggests that multiple sources and/or extended components contribute to the water emission from this star-forming region.

\section{Observations}

We made a deep integration towards W3 IRS5 with Odin from 2002 Jan. 25 to 2002 Jan. 28 with a total on-source integration time of $380 \mathrm{~min}$. During this period, we also obtained spectra of a 9 point map with $2^{\prime}$ spacing and typical integration times of 31 to 37 min per point. We subsequently made a 25 point 


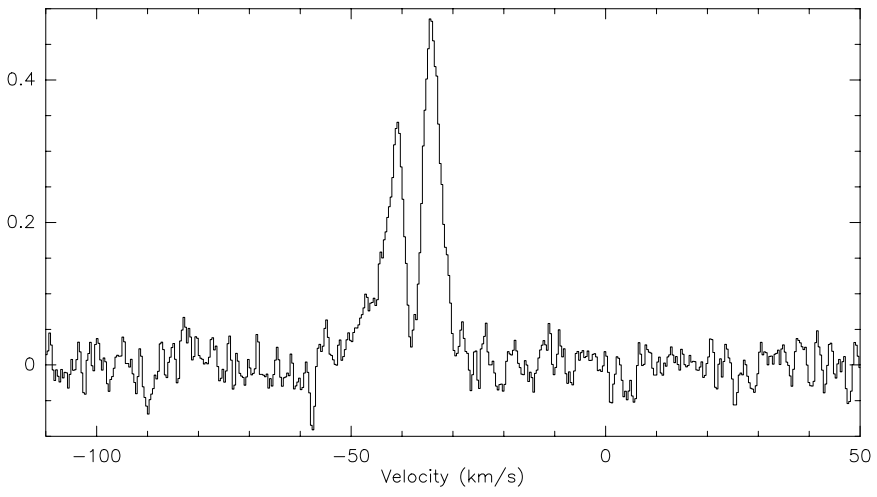

Fig. 2. Deep $\mathrm{H}_{2} \mathrm{O}$ integration towards W3 IRS5. The spectrum was obtained at 02:25:40.6+62:05:57 (J2000). The blue peak is $0.48 \pm$ $0.02 \mathrm{~K}\left(T_{A}^{*}\right)$ and the red peak is $0.34 \mathrm{~K}$ at a resolution of $1 \mathrm{MHz}$ $\left(0.54 \mathrm{~km} \mathrm{~s}^{-1}\right)$.

map with $1^{\prime}$ spacing from 2002 Mar. 11 to 2002 Mar. 19. The typical integration time was 36 min per point, with a minimum time of $33 \mathrm{~min}$ and a maximum time of $58 \mathrm{~min}$. All coordinates in this paper have been corrected for a global pointing offset of $\left(35^{\prime \prime},-10^{\prime \prime}\right)$ in January 2002 and $\left(34^{\prime \prime}, 13^{\prime \prime}\right)$ in March 2002, as determined from a map of Jupiter made in April 2002. The Odin beam at this frequency is $2.1^{\prime}$ and the typical system temperature was $3300 \mathrm{~K}$ (SSB). For all observations of W3, the spectrometer was configured with the acousto-optic spectrometer (AOS) and one autocorrelator observing the $556.936 \mathrm{GHz}$ line of ortho $-\mathrm{H}_{2} \mathrm{O}$ using two independent receivers. All observations were obtained in sky-switching mode and observations of a reference off position 30' east of W3 were obtained as well.

The AOS data were reduced using CLASS. To identify individual spectra which were contaminated by the Earth's atmosphere, we measured the average emission in a $60 \mathrm{~km} \mathrm{~s}^{-1}$ region around $0 \mathrm{~km} \mathrm{~s}^{-1}$ and removed spectra that were significantly above or below the average value. Spectra that were mis-tuned or that had anomalous system temperatures were also removed. The spectra for the off position were averaged and then smoothed with a gaussian function with a velocity full-width half-maximum of $1 \mathrm{~km} \mathrm{~s}^{-1}$. This smoothed spectrum was subtracted from each individual source spectrum to correct for the intrinsic fast ripple seen in the AOS spectra in sky-switching mode (Hjalmarson et al. 2003). Finally, all spectra within $20^{\prime \prime}$ of a given position were averaged together. The spectra were then hanning smoothed and first to third order baselines were fit over the region shown in Fig. 2 and excluding a window around the line from -60 to $-20 \mathrm{~km} \mathrm{~s}^{-1}$ for spectra with detections of the water line. The deep integration on W3 IRS5 shown in Fig. 2 has not been hanning smoothed and has had a fourth order baseline removed. The rms noise in the deep spectrum is $0.024 \mathrm{~K}$ at a resolution of $1 \mathrm{MHz}\left(0.54 \mathrm{~km} \mathrm{~s}^{-1}\right)$, while the rms noise in the individual spectra in the map ranges from 0.056 to $0.085 \mathrm{~K}$ at a resolution of $1.24 \mathrm{MHz}\left(0.67 \mathrm{~km} \mathrm{~s}^{-1}\right)$.

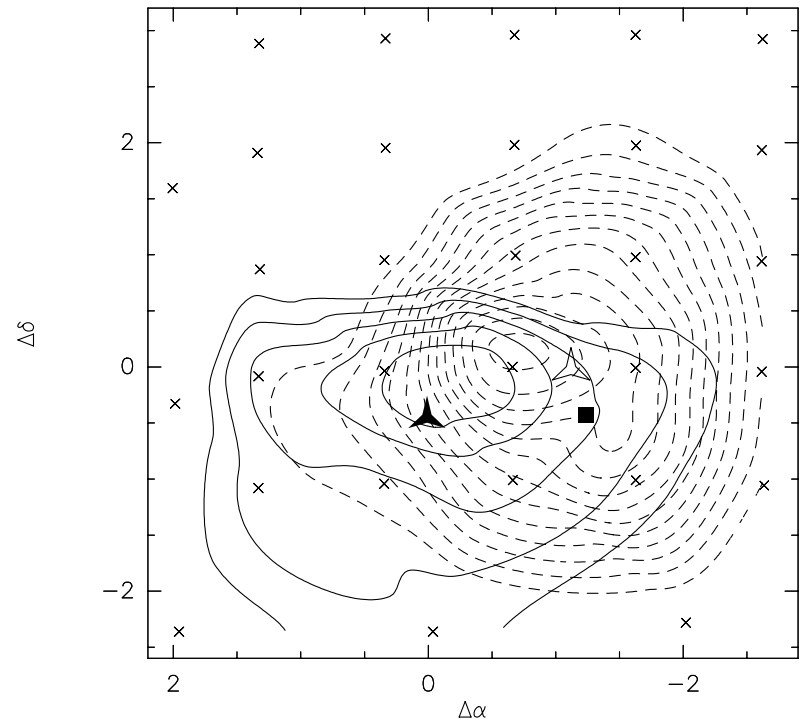

Fig. 3. Integrated intensity of the red $\left(-39\right.$ to $-28 \mathrm{~km} \mathrm{~s}^{-1}$, solid contours) and blue ( -52 to $-39 \mathrm{~km} \mathrm{~s}^{-1}$, dashed contours) components of the water lines in $\mathrm{W} 3$. Contours start at $0.75 \mathrm{~K} \mathrm{~km} \mathrm{~s}^{-1}$ and increase in steps of $0.25 \mathrm{~K} \mathrm{~km} \mathrm{~s}^{-1}(\sim 1 \sigma)$. The positions of W3 IRS5 and IRS4 are marked with filled and open pinwheels, respectively, and the SWAS pointing is marked with the filled square.

\section{Extended water emission towards W3 Main}

The relative strengths of the SWAS (Snell et al. 2000a) and Odin $\mathrm{H}_{2} \mathrm{O}$ detections suggest that the water emission in this region of W3 does not originate in a single, very compact source. The beam area of SWAS $\left(3.3^{\prime} \times 4.5^{\prime}\right)$ is 3.3 times larger than that of Odin at this frequency, yet the peak line strength measured by Odin in the W3 map is only 2.1 times stronger than the line measured by SWAS. In addition, the water emission observed with Odin appears significantly extended relative to the beam, with the red and blue components having different spatial distributions (Fig. 3). The observed full-width half-maximum diameter is $2.6^{\prime} \times 2.4^{\prime}$, which suggests a true diameter of $1.4^{\prime} \times \sim 1.2^{\prime}$. Individually, the red and blue components of the line are also significantly larger than the beam. Either the water emission in this region is significantly extended, or there are two or more strong point sources contained within the SWAS and Odin beams.

Two obvious candidates for point sources of water emission are W3 IRS5 and IRS4, which are separated by $\sim 70^{\prime \prime}$. However, the pointings of the SWAS spectrum and the strongest Odin spectrum relative to the locations of the two sources are such that the expected line strength ratio between the two satellites would still be about 3 if both sources are point-like. If the water emission seen in this region is associated with W3 IRS5 and IRS4, one or both of the sources must be significantly extended relative to the Odin beam. $\mathrm{C}^{18} \mathrm{O}$ mapping by Tieftrunk et al. (1995) reveals two molecular cores in this region, one $20^{\prime \prime} \times 20^{\prime \prime}$ associated with W3 IRS5 and a second $40^{\prime \prime} \times 30^{\prime \prime}$ core which lies slightly to the south of W3 IRS4 and shares the same central velocity. It seems likely that these molecular cores may account at least in part for the extent of the water emission in the $\mathrm{W} 3$ region. 


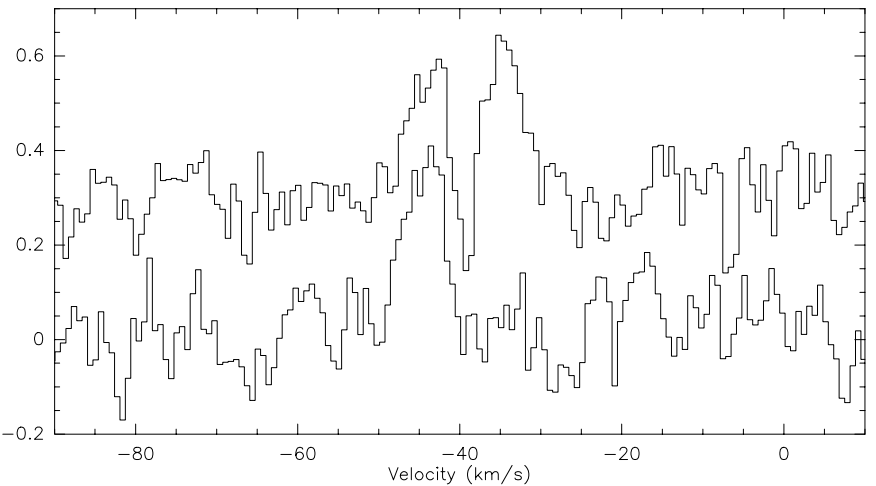

Fig. 4. Comparison of water spectra near W3 IRS5 (top) and W3 IRS4 (bottom). The vertical scale is -0.2 to $0.7 \mathrm{~K}$ and the W3 IRS5 spectrum has been offset by $0.3 \mathrm{~K}$ for clarity. Note the alignment in velocity between the blue wing of the W3 IRS5 spectrum and the peak of the W3 IRS4 spectrum.

The center of the absorption feature in the $\mathrm{H}_{2} \mathrm{O}$ spectra lies at $\sim-39 \mathrm{~km} \mathrm{~s}^{-1}$ across the map (see Fig. 2). The location of this absorption feature matches the central velocity of $\mathrm{NH}_{3}$ emission from W3 IRS5 quite well, and so towards this region we are likely seeing strongly self-absorbed water lines, similar to the $\mathrm{CO} J=3-2$ and $J=6-5$ lines observed by Hasegawa et al. (1994). However, the velocity towards W3 IRS4 and the molecular core just south of it is significantly different $\left(-42.5 \mathrm{~km} \mathrm{~s}^{-1}\right.$, Tieftrunk et al. 1998; Hasegawa et al. 1994). Thus, it is difficult to understand the constant velocity of the $-39 \mathrm{~km} \mathrm{~s}^{-1}$ absorption feature across the map given the velocity shift of $3-$ $4 \mathrm{~km} \mathrm{~s}^{-1}$ that is seen in optically thin tracers such as $\mathrm{C}^{18} \mathrm{O}$ and $\mathrm{NH}_{3}$ moving from west (near W3 IRS4) to east (W3 IRS5) across the region (Tieftrunk et al. 1995, 1998).

The most likely possibility is that we are seeing water emission from two distinct regions in our map, one associated with W3 IRS5 and the second associated with W3 IRS4 and/or its molecular core. In this model, it is the coincidental agreement in velocity between the single-peaked line from W3 IRS4 and the blue-shifted peak of the self-absorbed spectrum from the W3 IRS5 region that produces the constant velocity of the absorption feature at $-39 \mathrm{~km} \mathrm{~s}^{-1}$. This interpretation is illustrated in Fig. 4, which compares the observed spectra near W3 IRS5 and W3 IRS4. The W3 IRS5 region contains at least two outflows (Hasegawa et al. 1994; Imai et al. 2000), which could be partly responsible for the prominence of the red-shifted emission peak. The second region is associated with W3 IRS4 itself (and its associated photon dominated region) and/or the extended molecular core near W3 IRS4 (Tieftrunk et al. 1995, 1998). If water emission from this region is not self-absorbed, we would expect the emission to peak at a velocity of -42 to $-43 \mathrm{~km} \mathrm{~s}^{-1}$, in reasonable agreement with our observations.

We have estimated the $\mathrm{H}_{2} \mathrm{O}$ abundance towards W3 IRS5 using the formula given by Snell et al. (2000b). We use a density of $1 \times 10^{6} \mathrm{~cm}^{-3}$ (Snell et al. 2000a) and adopt a temperature of $40 \mathrm{~K}$. We estimate an $\mathrm{H}_{2}$ column density of $5 \times 10^{22} \mathrm{~cm}^{-2}$ inside the Odin beam from the mass of $1900 M_{\odot}$ derived for the W3 core (Tieftrunk et al. 1995, scaled to a temperature of $40 \mathrm{~K}$ ). The integrated intensity of $3.9 \pm 0.1 \mathrm{~K} \mathrm{~km} \mathrm{~s}^{-1}$ (Fig. 2) results in an ortho- $\mathrm{H}_{2} \mathrm{O}$ abundance of $2 \times 10^{-9}$, in excellent agreement with the results from SWAS (Snell et al. 2000a). We will present a more detailed analysis of the $\mathrm{W} 3$ region, including follow-up $\mathrm{H}_{2}^{18} \mathrm{O}$ and $\mathrm{NH}_{3}$ observations, in a future paper.

Acknowledgements. Generous financial support from the Research Councils and Space Agencies in Canada, Finland, France, and Sweden is gratefully acknowledged.

\section{References}

Bally, J., \& Lada, C. J. 1983, ApJ, 265, 824

Bergin, E. A., Melnick, G. J., Stauffer, J. R., et al. 2000, ApJ, 539, L129

Frisk, U., Hagström, M., Ala-Laurinaho, J., et al. 2003, A\&A, 402, L27

Goldsmith, P. F., \& Langer, W. D. 1978, ApJ, 222, 881

Hasegawa, T. I., Mitchell, G. F., Matthews, H. E., \& Tacconi, L. 1994, ApJ, 426, 215

Helmich, F. P., Jansen, D. J., de Graauw, Th., Groesbeck, T. D., \& van Dishoeck, E. F. 1994, A\&A, 283, 626

Helmich, F. P., \& van Dishoeck, E. F. 1997, A\&AS, 124, 205

Hjalmarson, A., Frisk, U., Olberg, M., et al. 2003, A\&A, 402, L39

Imai, H., Kameya, O., Sasao, T., et al. 2000, ApJ, 538, 751

Megeath, S. T., Herter, T., Beichmann, C., et al. 1996, A\&A, 307, 775

Neufeld, D. A., Snell, R. L., Ashby, M. L. N., et al. 2000, ApJ, 539, L107

Nordh, L., von Scheele, F., Frisk, U., et al. 2003, A\&A, 402, L21

Snell, R. L., Howe, J. E., Ashby, M. L. N., et al. 2000a, ApJ, 539, L101

Snell, R. L., Howe, J. E., Ashby, M. L. N., et al. 2000b, ApJ, 539, L93

Tieftrunk, A. R., Wilson, T. L., Steppe, H., et al. 1995, A\&A, 303, 901

Tieftrunk, A. R., Gaume, R. A., Claussen, M. J., Wilson, T. L., \& Johnston, K. J. 1997, A\&A, 318, 931

Tieftrunk, A. R., Megeath, S. T., Wilson, T. L., \& Rayner, J. T. 1998, A\&A, 336, 991

van Dishoeck, E. F., \& Helmich, F. P. 1996, A\&A, 315, L177

Wynn-Williams, C. G., Becklin, E. E., \& Neugebauer, G. 1972, MNRAS, 160, 1 